\title{
Anticoncepción como prevención de complicaciones por enfermedades crónicas asociadas al embarazo.
}

\author{
Anticonception as prevention of complications for chronic diseases associated \\ with pregnancy.
}

Nancy Corina Sánchez Coronel. ${ }^{1}$, Gabriel José Suarez Lima. ${ }^{2}$, Nixon Federico Holguín Salazar. ${ }^{3}$ \& Laura Judith Zuñiga Fariño. ${ }^{4}$

Recibido: 14-03-2020 / Revisado: 19-04-2020 /Aceptado: 05-05-2020/ Publicado: 05-06-2020

\begin{abstract}
.
DOI: https://doi.org/10.33262/concienciadigital.v3i2.2.1243

I Introduction: Chronic diseases are long-term illnesses, according to the WHO indicated that 830 women die every day worldwide due to chronic diseases related to pregnancy or childbirth, being considered one of the public health problems. Objective. Identify contraceptive methods as prevention in complications due to chronic diseases associated with pregnancy in patients of the Teodoro Maldonado Carbo Hospital from 2018 - 2019. Method. The design and type of the research was quantitative, retrospective, transversal and descriptive, the population was 83 cases, while the sample was chosen 47 cases of pregnant women with a diagnosis of chronic diseases, between May 2018 to May 2019 As an instrument, a data collection sheet validated by expert judgment was used. Results The most commonly used contraceptive methods were injectable methods (21.3\%), pills (14.9\%), age range; from 35 to 44 years (46.8\%), the level of instruction; secondary (48.9\%), ethnic group; mestizo (44.7\%), place of residence; Guayaquil (44.7\%), Chronic disease, Chronic renal failure on hemodialysis $12.8 \%$ as well as chronic arterial hypertension, Frequent pregnancy number; 3rd pregnancy (34\%), years of suffering with the clinical condition; 0 to 3 years $(63.8 \%)$, medical complication; HELLP syndrome (38.3\%), use of contraceptive methods recommended by the attending physician; if $(27.7 \%)$ and not $(72.3 \%)$. Conclusion. The population studied recognizes that the level of knowledge about contraceptive methods was regular, since many did not apply it and if they used it improperly.
\end{abstract}

Keywords: Contraceptive methods, chronic diseases, pregnant women, complications in pregnant women.

\section{Resumen}

Introducción: Las enfermedades crónicas son padecimientos de larga duración, según la OMS indicó que cada día mueren a nivel mundial 830 mujeres por enfermedades crónicas

\footnotetext{
${ }^{1}$ Universidad Estatal de Milagro, Milagro, Ecuador, nsanchezc@unemi.edu.ec

${ }^{2}$ Universidad Estatal de Milagro, Milagro, Ecuador, Facultad Ciencias de la Salud, gsuarezl@unemi.edu.ec

${ }^{3}$ Doctor Traumatólogo Ortopedista, Milagro, Ecuador, drniholsa@ hotmail.com

${ }^{4}$ Universidad Estatal de Milagro, Facultad Ciencias de la Salud, Milagro, Ecuador, laurazfario@hotmail.com
} 
relacionadas con el embarazo o el parto, considerándose uno de los problemas de salud pública. Objetivo. Identificar los métodos anticonceptivos como prevención en las complicaciones por enfermedades crónicas asociadas al embarazo en las pacientes del Hospital Teodoro Maldonado Carbo del 2018 - 2019. Método. El diseño y tipo de la investigación fue cuantitativa, de tipo retrospectiva, transversal y descriptiva, la población fue de 83 casos, mientras que la muestra se escogieron 47 casos de mujeres embarazadas con diagnóstico de enfermedades crónicas, entre mayo del 2018 a mayo del 2019, como instrumento se utilizó una ficha de recolección de datos validada por juicio de expertos. Resultados. Los métodos anticonceptivos mayormente utilizados fueron los métodos inyectables (21,3\%), pastillas (14,9\%), rango de edad; de 35 a 44 años (46,8\%), el nivel de instrucción; secundaria (48,9\%), grupo étnico; mestiza (44,7\%), Lugar de residencia; Guayaquil (44,7\%), Enfermedad crónica, Insuficiencia renal crónica en hemodiálisis $12,8 \%$ al igual que la Hipertensión arterial crónica, Número de embarazo frecuente; 3er embarazo (34\%), años de padecimiento con la condición clínica; 0 a 3 años (63,8\%), Complicación médica; Síndrome HELLP $(38,3 \%)$, uso de métodos anticonceptivos recomendado por el médico tratante; si el $(27,7 \%)$ y no el $(72,3 \%)$. Conclusión. La población estudiada, reconoce que el nivel de conocimiento sobre los métodos anticonceptivos fue regular, ya que muchas no lo aplicaban y si lo empleaba de la forma inadecuada.

Palabras claves: Métodos anticonceptivos, enfermedades crónicas, mujeres embarazadas, complicaciones en gestantes.

\section{Introducción}

La carga para los sistemas de salud, causada por el aumento de las enfermedades crónicas (EC), constituye uno de los principales desafíos globales para el desarrollo en el siglo XXI. Para disminuir el gasto sanitario por motivos evitables, se precisa reducir la mortalidad prematura, mejorar la calidad de la vida de la población y conceder prioridad a la prevención y el control de dichas patologías. Formando parte del listado de problemas que irrumpen el proceso saludenfermedad y provocan complicaciones asociadas al embarazo, se ubica una inadecuada anticoncepción.

La necesidad de controlar las familias numerosas y disminuir los riesgos a la morbilidad en mujeres con EC, constituyen un problema de salud pública no resuelto, el que deviene desde el surgimiento de la historia de la humanidad (Pozuelos Mendoza, 2018).

El origen de la anticoncepción y con esta, el control de la natalidad se remonta al surgimiento de la vida y al descubrimiento de la asociación entre la práctica de las relaciones sexuales y la concepción del embarazo en mujeres fértiles; elemento que debe ser tomado en consideración por la población en general; con énfasis por aquellas féminas en las que coexiste una EC que condiciona la salud del organismo materno y compromete la fetal. Para alcanzar lo antes dicho, se precisa que la pareja y en especial la mujer, alcancen una adecuada salud sexual y reproductiva (SSR); comportamiento determinado, entre otros elementos por la planificación familiar (PF), método empelado desde el desarrollo de la humanidad; el cual, como cualquier otro aspecto de carácter sociocultural, puede estar influenciado por condicionantes que la favorecen o entorpecen. (Huerta, Sáinz Gómez, \& Antonio Solís, 2014)

La mayoría de los embarazos y de los partos transcurren sin incidentes, sin embargo, representan un riesgo. Se estima que alrededor del $15 \%$ del total de las mujeres embarazadas manifiestan complicaciones totalmente fatales que requieren de atención inmediata y en 
algunos casos, una intervención obstétrica importante para que sobrevivan. (Gómez Sánchez, Gaitán Duarte, \& Rodríguez Malagón, 2017)

Según la (OMS, 2016) cada día mueren en todo el mundo unas 830 mujeres por complicaciones relacionadas con el embarazo o el parto. En 2015 se estimaron unas 303000 muertes de mujeres durante el embarazo y el parto o después de ellos, 2,7 millones de niños fallecieron durante los primeros 28 días de vida y la cifra de mortinatos alcanzó los 2,6 millones. La atención sanitaria de calidad durante el embarazo y el parto puede prevenir muchas de esas muertes.

Alcanzar una apropiada SSR demanda de conocimientos por parte de la mujer, lo que a su vez contribuye a evitar fracaso en el tratamiento anticonceptivo y garantizar incremento de la eficacia de estos; disminuyendo consigo la instauración de efectos secundarios y problemas de salud derivados de su empleo inadecuado. (Calixto Morales, Ospina Díaz, \& Manrique Abril, 2015)

El incremento del número de embarazos no planificados, con énfasis en la adolescencia, la realización de abortos inseguros y la baja adherencia de las mujeres con EC al régimen terapéutico requerido por su patología de base, contribuyen al aumento de la mortalidad materna y perinatal; lo cual, desde un análisis salubrista, expresa la necesidad de incrementar acciones dirigidas a educar a la población diana para revertir la situación derivada de la baja percepción del riesgo de complicaciones y la práctica de tratamiento anticonceptivo de forma no segura. (Adarve Hidalgo, Falguera, \& Seguranyes, 2016)

La situación antes expuesta repercute en el éxito que la anticoncepción persigue, realidad de la cual no escapa Ecuador, país pluricultural en el que se observa altos índice de embarazo en la adolescencia, en mujeres con EC y de gestaciones no deseadas.

En Ecuador, según datos ofrecidos por la Encuesta Nacional de Salud y Nutrición (Ministerio de Salud Pública, 2017) la población global estimada para el año 2016 fue de 16,613.279 Personas; de la cual el 55,5\% correspondió al sexo femenino; cifra mayoritariamente constituida por mujeres en edad fértil, en las que, en correspondencia con la literatura científica citada y las estadísticas consultadas, coexiste comorbilidad por EC, en algunas pacientes de forma diagnosticadas y en otras en curso de manera inadvertida o inaparente. (Ministerio de Salud Pública, 2017) (INEC, 2018) (Braganza Guananga, 2016)

La última década acaecida en este contexto, muestra un incremento de partos en adolescentes, cuyas edades oscilan entre 12 y 19 años. Ámbito en el que según datos históricos del MSP (2018), en el país se registran 413,318 nacidos vivos de niñas y adolescentes, evidencia que demuestra la necesidad de adoptar medidas enfocadas en la yugulación de esa problemática. (Mera Zambrano, Roca Castro, \& Medranda Obregón, 2016)

En concordancia con esos resultados, el Instituto Nacional de Estadísticas y Censos (INEC) confirmó que el 19,6\% de los nacimientos ocurridos en el año 2017 provinieron de madres con esas edades, realidad que demuestra que aún los resultados del Plan Nacional de Salud Sexual y Salud Reproductiva 2017 - 2021 implementado por el MSP no alcanza a cumplir con la meta relativa al desarrollo de estrategias dirigidas a la modificación de los indicadores de impacto negativo en este sector poblacional, responsables de las elevadas cifras de parto y muerte materna e infantil. (INEC, 2018) (Ministerio de Salud Pública, 2017)

En relación a la necesidad de desarrollar acciones estratégicas basadas en la prevención de complicaciones asociadas al embarazo causadas por EC, la comunidad científica posiciona a 
la educación sanitaria en materia de SSR como elemento clave, y recomienda que previo a la indicación del uso de algún método anticonceptivo, la pareja o de no ser posible entrevistar a ambos, la mujer con vida sexual activa debe ser clínicamente estudiada, para que de esa forma el binomio pueda emplear la forma de anticoncepción que mejor se adapten a ambos; razón por la que los individuos de ambos sexos en edad reproductiva, deben tener la posibilidad de acceder, elegir y beneficiarse de los avances científicos generados de la variedad anticonceptiva existente. (Escobar González, 2016)

En el contexto ecuatoriano, lo anterior induce a la adopción de estrategias nacionales e institucionales dirigidas a la capacitación de los profesionales de la salud que laboran en atención directa a este tipo de población, con el objetivo de fomentar la direccionalidad técnica de ese proceso, como recurso para mitigar las consecuencias derivadas de una inadecuada PF y por consiguiente deficiente SSR en la edad fértil y para paliar la autoprescripción anticonceptiva. (Ministerio de Salud Pública, 2017)

En relación a este último particular, estudios efectuados por Adarve en el 2016 plantea que la adopción de una contracepción no consultada a un especialista en el tema, deriva en la aparición de complicaciones en la mujer, con énfasis en la portadora de EC. Estudios sobre esta temática reconocen una relación directamente proporcional en el logro de resultados positivos entre la efectividad de la anticoncepción femenina prescrita mediante asesoría reproductiva y el rol del personal de salud en la labor educativa sobre este particular. (Adarve Hidalgo, Falguera, \& Seguranyes, 2016)

En el ámbito de desarrollo de la investigación que se presenta, Hospital Teodoro Maldonado Carbo de la ciudad de Guayaquil, los resultados estadísticos evidencian que entre las patologías mayormente reportadas en pacientes con EC, se encuentran en orden decreciente de casos, las de causa respiratoria, cardiovascular, renal, endocrino metabólicas, neoplásicas, del colágeno e inmunológicas, con un período de padecimiento entre uno y tres años de diagnosticadas.

Tomando en consideración lo antes descrito y el criterio de que la prescripción de anticonceptivos acorde a la problemática de cada paciente reduce la posibilidad de embarazos en mujeres cuya enfermedad de base puede complicar el curso de la gestación; permite postergarlos para un momento en el que la patología este controlada y represente un menor riesgo para la salud y la vida de la mujer y el producto de su concepción, se ha considerado como necesario realizar el estudio que se presenta, como vía para ofrecer solución al problema de salud identificado.

Por lo tanto, el presente trabajo de investigación tiene como propósito de identificar los métodos anticonceptivos utilizados como prevención en las complicaciones por enfermedades crónicas asociadas al embarazo en las pacientes del Hospital Teodoro Maldonado Carbo del $2018-2019$.

\section{Método}

El diseño y tipo de la investigación fue cuantitativa, de tipo retrospectiva, transversal y descriptiva, solo a mujeres embarazadas con EC en el Hospital Teodoro Maldonado Carbo, entre mayo del 2018 y mayo del 2019, la población fue de 83 casos, mientras que la muestra se escogieron 47 casos cumpliendo el criterio de inclusión solo con mujeres embarazadas con diagnóstico de enfermedades crónicas, los datos fueron tomados directamente de las historias clínicas del Sistema AS400 con el permiso de la máxima autoridad al director administrativo del hospital. 
Como instrumento se utilizó una ficha de recolección de datos validada por juicio de expertos en salud pública, el cual constó con 10 ítems siendo los mismos validados por juicios de expertos calificando validez, pertinencia y coherencia, donde la validez alcanzó una calificación del 98\%, la pertinencia un 94\%, y confiabilidad un $96 \%$.

Para recabar información de interés investigativo referente a las características sociodemográficas y clínicas de las mujeres incluidas en el estudio $(n=47)$ se revisaron las historias clínicas de las mismas, instrumento que permitió conocer sobre la edad, nivel educacional, estado civil, ocupación, nivel de conocimiento sobre anticoncepción, tipo de anticoncepción empleada, tiempo de uso de la misma, diagnóstico de enfermedad crónica.

El instrumento constó con los siguientes ítems; Métodos anticonceptivos utilizados; (Pastillas - Inyectables - Implantes - Dispositivos - Histerectomía - Ligadura de trompas - Condón femenino - Diagrama Vaginal - Ninguno.), Grupo etario (Menor a 18 - 18 años a 24 años - 25 años a 34 años - 35 años a 44 años - 45 años a 54 años - más de 54 Años), Nivel de instrucción; (Sin formación - Primaria - Secundaria - Universidad - Cuarto Nivel), Grupo étnico (Mestiza - Afroecuatoriana - Blanca .montubia - Indígena), Lugar de Residencia (La Troncal Guayaquil - Balzar - Tenguel - Huaquillas), Enfermedades crónicas (Insuficiencia Renal Crónica - IRC en Hemodiálisis - HTP - HTA - DM2 - Leucemias promelocitica - LES - EPOC - AR - Trastornos Mentales - Trombocitopenia - Trombosis venosa profunda - Hepatopatía crónica - Insuficiencia mitral severa - Linfoma no Hoogkin - Estenosis aortica severa Epilepsia refractaria - Síndrome Antifosfolipídico - Displasia arritmogenica del VD - Tumor Renal metastásico - Cáncer de cabeza de páncreas - Malformación arterio venosa de vasos cerebrales - Cancer de cervix - Litiasis renal bilateral - Otras Infarto cerebral, síndrome convulsivo) Número de embarazos con la actual condición clínica crónica; $\left(1^{\circ}\right.$ Embarazo - $2^{\circ}$ Embarazo - $3^{\circ}$ Embarazo - $4^{\circ}$ Embarazo - $5^{\circ}$ Embarazo - $6^{\circ}$ Embarazo), Años de padecimiento con condición clínica crónica; (0 a 3 Años - 4 a 7 Años - 8 a 11 Años - 12 a 15 años - >16 años), Complicaciones que presentaron las mujeres durante el embarazo (Eclampsia Preeclampsia Severa - Síndrome HELLP - Insuficiencia respiratoria - Aborto incompleto Neumonía - Urosepsis - Pancreatitis Biliar más estenosis biliar - Shock Séptico), El uso de métodos anticonceptivos se lo recomendó el médico que atiende su patología crónica ( $\mathrm{Si}-\mathrm{No}$ ).

\section{Resultados}

Fue de suma importancia analizar los datos sociodemográficos el cual se dio a conocer la distribución de mujeres en edad según escala de rangos, las edades comprendidas entre los 35 a 44 años con el 46,61\%, fue el rango que más abarcó en el estudio, seguido del 31,91\% en edades comprendidas de 25 a 34 años, luego con 19,15\% en edades comprendidas 15 a 24 años y con el $2,13 \%$ en edades de 45 a 54 años. Se constató el nivel de instrucción de la paciente embarazada, donde se observó 23 casos de nivel secundaria equivalente a un 48,9\%, seguido de 11 casos de nivel primaria correspondiente a un 23,4\%, 6 casos de pacientes embarazadas en universidad formando un porcentaje del 12,8\%, 5 sin formación representado un 10,6\%, y 2 de ellas con título de cuarto nivel reflejando un 4,3\%. El grupo étnico de las mujeres embarazadas, se reflejó 21 casos de pacientes mestiza correspondiente a un 44,7\%, 8 casos de raza blanca equivalente a un $17 \%$ al igual que raza montubia, 6 casos fueron de etnia indígena perteneciente a un $12,8 \%$, y 4 casos Afroecuatoriana formando parte de un 8,5\%. Con respecto al lugar de residencia de las mujeres embarazada, Guayaquil tiene el 44,68 de pacientes con procedencia de enfermedades crónicas, seguido de La Troncal con el 19,15\%, Balzar el 8,51\%, Tenguel el 10,64\%, Huaquillas y Sto. Domingo el 8,51\%, tal como se detalla en la siguiente tabla $\mathrm{N}^{\circ} 1$. 
Tabla 1. Características sociodemográficas de las mujeres embarazadas del HTMC.

Rango de edades de las pacientes

\begin{tabular}{|c|c|c|}
\hline & Frecuencia & Porcentaje \\
\hline De 15 a 24 años & 9 & $19,10 \%$ \\
\hline De 25 a 34 años & 15 & $31,90 \%$ \\
\hline De 35 a 44 años & 22 & $46,80 \%$ \\
\hline De 45 a 54 años & 1 & $2,10 \%$ \\
\hline Total & 47 & $100 \%$ \\
\hline \multicolumn{3}{|l|}{ Nivel de Instrucción } \\
\hline Sin Formación & 5 & $10,60 \%$ \\
\hline Primaria & 11 & $23,40 \%$ \\
\hline Secundaria & 23 & $48,90 \%$ \\
\hline Universidad & 6 & $12,80 \%$ \\
\hline Cuarto Nivel & 2 & $4,30 \%$ \\
\hline Total & 47 & $100 \%$ \\
\hline \multicolumn{3}{|l|}{ Grupo Étnico } \\
\hline Mestiza & 21 & $44,70 \%$ \\
\hline Afroecuatoriana & 4 & $8,50 \%$ \\
\hline Blanca & 8 & $17,00 \%$ \\
\hline Montubia & 8 & $17,00 \%$ \\
\hline Indígena & 6 & $12,80 \%$ \\
\hline Total & 47 & $100 \%$ \\
\hline \multicolumn{3}{|l|}{ Lugar de Residencia } \\
\hline La Troncal & 9 & $19,10 \%$ \\
\hline Guayaquil & 21 & $44,70 \%$ \\
\hline Balzar & 4 & $8,50 \%$ \\
\hline Tenguel & 5 & $10,60 \%$ \\
\hline Huaquillas & 4 & $8,50 \%$ \\
\hline Sto. Domingo & 4 & $8,50 \%$ \\
\hline Total & 47 & $100 \%$ \\
\hline
\end{tabular}

Elaborado: por la Dra Nancy Sánchez Coronel

Fue considerado de suma importancia saber las enfermedades crónicas que presentaron dichas pacientes embarazadas, el cual 6 de las pacientes reflejaron Insuficiencia Renal Crónica en Hemodiálisis correspondiente a un 12,8\%, al igual que la HTA Crónica, seguida de la IRC con 4 casos equivalente a un $8,5 \%$, de la misma manera la HTP con la misma frecuencia, la insuficiencia mitral severa con 3 casos formando un 6,4\%, la DM2, LES, EPOC, AR cada una con 2 casos en pacientes presentando así un 4,3\%, con respecto a la leucemia, trastornos mentales, trombocitopenia, trombosis venosa, hepatopatía crónica, linfoma no Hoogkin, estenosis aortica severa, epilepsia refractaria, síndrome Antifosfolipídico, displasia arritmogenica del VD, tumor renal metastásico, cáncer de cabeza de páncreas, malformación arterio venosa de vasos cerebrales, cáncer de cervix, litiasis renal bilateral, e infarto cerebral, síndrome convulsivo, donde cada una de estas enfermedades se presentan en cada paciente, como se refleja en la tabla $\mathrm{N}^{\circ} 2$. 
Tabla 2. Enfermedad Crónica que presentaron las pacientes embarazadas

\begin{tabular}{lcc}
\hline Enfermedades Crónicas & Frecuencia & Porcentaje \\
\hline Insuficiencia Renal Crónica & $\mathbf{4}$ & $8,50 \%$ \\
IRC en Hemodiálisis & $\mathbf{6}$ & $12,80 \%$ \\
HTP & 4 & $8,50 \%$ \\
HTA Crónica & 6 & $12,80 \%$ \\
DM2 & 2 & $4,30 \%$ \\
Leucemias promelocitica & 1 & $2,10 \%$ \\
LES & 2 & $4,30 \%$ \\
EPOC & 2 & $4,30 \%$ \\
AR & 2 & $4,30 \%$ \\
Trastornos Mentales & 1 & $2,10 \%$ \\
Trombocitopenia & 1 & $2,10 \%$ \\
Trombosis venosa profunda & 1 & $2,10 \%$ \\
Hepatopatía crónica & 1 & $2,10 \%$ \\
Insuficiencia mitral severa & 3 & $6,40 \%$ \\
Linfoma no Hoogkin & 1 & $2,10 \%$ \\
Estenosis aortica severa & 1 & $2,10 \%$ \\
Epilepsia refractaria & 1 & $2,10 \%$ \\
Síndrome Antifosfolipídico & 1 & $2,10 \%$ \\
Displasia arritmogenica del VD & 1 & $2,10 \%$ \\
Tumor Renal metastásico & 1 & $2,10 \%$ \\
Cáncer de cabeza de páncreas & 1 & $2,10 \%$ \\
Malformación arterio venosa & 1 & $2,10 \%$ \\
vasos cerebrales & 1 & $2,10 \%$ \\
Citiasis renal bilateral & 1 & $2,10 \%$ \\
Cotras (Infarto cerebral, síndrome & $100,00 \%$ \\
\hline
\end{tabular}

Elaborado: por la Dra Nancy Sánchez Coronel

Se constató en número de embarazos de las mujeres, donde la gran mayoría fue su tercer embarazo abarcando un porcentaje del $34,04 \%$, seguido del cuarto embarazo con un $23,40 \%$, en el segundo embarazo un $21,28 \%$, en el primer embarazo el $19,15 \%$, mientras que en el sexto embarazo fue un $2,13 \%$. Además se determinó el año de padecimiento de las pacientes con la enfermedades crónicas el 63,83\% corresponden de 0 a 3 años, el 27,66\% corresponde entre 4 a 7 años y, con el 6,38\% respectivamente entre edades de 8 a 11 años y el 2,13\%, de 12 a 15 años. Con respecto a las complicaciones médicas más presentadas en las pacientes reflejaron 18 casos con síndrome HELLP equivalente a un 38,3\%, 15 casos por preeclampsia severa correspondiente a un 31,9\%, 4 casos con insuficiencia respiratoria abarcando un porcentaje de 8,5\%, 3 casos con aborto incompleto efectuando un 6,4\% del total del estudio, la eclampsia y la neumonía cada una de ellas presentaron 2 casos formando parte de un 4,3\%, la urosepsis, pancreatitis Biliar más estenosis biliar y shock séptico cada una de ellas con 1 casos a cada paciente el cual refleja un $2,1 \%$, tal como se presenta en la tabla $\mathrm{N}^{\circ} 3$. 
Tabla 3. Número de embarazo, años de padecimiento y complicaciones médicas

\begin{tabular}{|c|c|c|}
\hline \multicolumn{3}{|c|}{ Número de embarazo con padecimiento de enfermedades crónicas } \\
\hline & Frecuencia & Porcentaje \\
\hline $1^{\circ}$ Embarazo & 9 & $19,10 \%$ \\
\hline $2^{\circ}$ Embarazo & 10 & $21,30 \%$ \\
\hline $3^{\circ}$ Embarazo & 16 & $34,00 \%$ \\
\hline $4^{\circ}$ Embarazo & 11 & $23,40 \%$ \\
\hline $6^{\circ}$ Embarazo & 1 & $2,10 \%$ \\
\hline Total & 47 & $100,00 \%$ \\
\hline \multicolumn{3}{|l|}{ Años de padecimiento de enfermedades crónicas } \\
\hline 0 a 3 años & 30 & $63,80 \%$ \\
\hline 4 a 7 años & 13 & $27,70 \%$ \\
\hline 8 a 11 años & 3 & $6,40 \%$ \\
\hline 12 a 15 años & 1 & $2,10 \%$ \\
\hline Total & 47 & $100,00 \%$ \\
\hline \multicolumn{3}{|l|}{ Complicaciones médicas } \\
\hline Eclampsia & 2 & $4,30 \%$ \\
\hline Preeclampsia Severa & 15 & $31,90 \%$ \\
\hline Síndrome HELLP & 18 & $38,30 \%$ \\
\hline Insuficiencia respiratoria & 4 & $8,50 \%$ \\
\hline Aborto incompleto & 3 & $6,40 \%$ \\
\hline Neumonía & 2 & $4,30 \%$ \\
\hline Urosepsis & 1 & $2,10 \%$ \\
\hline Pancreatitis Biliar más estenosis biliar & 1 & $2,10 \%$ \\
\hline Shock Séptico & 1 & $2,10 \%$ \\
\hline Total & 47 & $100,00 \%$ \\
\hline
\end{tabular}

Elaborado: por la Dra Nancy Sánchez Coronel

En relación al uso de métodos anticonceptivos por parte del médico que atendió la patología, el $72,34 \%$, se reflejó en que no utilizaron, siendo esto un alto indice de responsabilidad médica ginecológica, mientras que el $27,66 \%$ si se observó recomendación del uso de métodos anticonceptivos por el médico, como se muestra en la tabla $\mathrm{N}^{\circ} 4$.

Tabla 4. Uso de métodos anticonceptivos recomendado por el médico que atendió la patología crónica

\begin{tabular}{lll}
\hline & Frecuencia & Porcentaje \\
\hline Si & 13 & $27,70 \%$ \\
No & 34 & $72,30 \%$ \\
Total & 47 & $100,00 \%$ \\
\hline
\end{tabular}

Elaborado: por la Dra. Nancy Sánchez Coronel 
El 29,79\% de las pacientes no han utilizado métodos anticonceptivos, sin embargos el 21,26\% ha empleado la inyección como método anticonceptivo alternativo, seguido del 14,89\% por uso de pastillas, el $12,77 \%$ por uso de dispositivos, el $8,51 \%$ por ligaduras de las trompas, el $6,38 \%$ por uso de implantes, el $4,26 \%$ por uso de condón vaginal y con el $2,13 \%$ por histerectomía, como se observa en la tabla $\mathrm{N}^{\circ} 5$.

Tabla 5. Métodos anticonceptivos utilizados por pacientes con enfermedades crónicas

\begin{tabular}{lcc}
\hline & Frecuencia & Porcentaje \\
\hline Pastillas & 7 & $14,90 \%$ \\
Inyectables & 10 & $21,30 \%$ \\
Implantes & 3 & $6,40 \%$ \\
Dispositivos & 6 & $12,80 \%$ \\
Histerectomía & 1 & $2,10 \%$ \\
Ligadura de trompas & 4 & $8,50 \%$ \\
Condón femenino & 2 & $4,30 \%$ \\
Ninguno & 14 & $29,80 \%$ \\
Total & 47 & $100,00 \%$ \\
\hline
\end{tabular}

Elaborado: por la Dra. Nancy Sánchez Coronel

\section{Discusión}

En el presente estudio se determinaron los datos sociodemográficos de las mujeres embarazadas con enfermedades en el cual en el rango de edad con mayor frecuencia fue entre los 35 a 44 años con el 46,61\%, el nivel de instrucción se observó 23 casos de nivel secundaria equivalente a un $48,9 \%$, el grupo étnico de las mujeres embarazadas fue mestiza con un 44,7\%, el cual la mayoría habitaba en zonas urbanas.

Según el estudio de Oscar Fawed Reyes en el año 2016 con el tema "Complicaciones Obstétricas en Adolescentes y Mujeres Adultas con o sin Factores de Riesgo Asociados, Honduras 2016", con estudio Analítico retrospectivo, realizado en el Hospital Escuela Universitario, Tegucigalpa, Honduras, la población fue de 380 expedientes de mujeres embarazadas, el cual identificó que el rango de edad frecuente fue de 20 a 35 años con un $33,68 \%$, el nivel máximo alcanzado en el nivel de estudio fue la secundaria con un $40,26 \%$, el grupo étnico fue mestiza con un $97,11 \%$ y de procedencia urbana con un $85,53 \%$. (Fawed Reyes, y otros, 2016)

Por lo tanto, en ambos estudios se pudo verificar que tienen un grado de similitud, tanto en el nivel de instrucción, grupo étnico y procedencia, a excepción de la variable del rango de edad ya que en el estudio de Fawed Reyes reflejo que las mujeres embarazadas en su estudio fue más frecuente el rango de edad de 20 a 35 años, mientras que en el del estudio presente fue de 35 a 44 años.

Adema en el presente estudio de identificó las enfermedades crónicas que más fueron presentadas en dichas pacientes embarazadas, en donde la Insuficiencia Renal Crónica en Hemodiálisis abarcó un 12,8\%, al igual que la HTA Crónica, y la IRC con un 8,5\%, siendo 
estas consideradas las más frecuentes en el estudio.

Según el trabajo investigativo de Wilmer Gerardo Rojas en el año 2015 con el tema "Enfermedad renal crónica y embarazo" realizado en Bogotá, Colombia, fue un estudio cuantitativo y descriptivo el cual identificó ocho casos de enfermedad renal crónica y embarazo, su presentación clínica y el desenlace obstétrico, dando a conocer que la Insuficiencia renal crónica es la que con mayor frecuencia presentó con un 26,32\%, seguida de la Preeclampsia, síndrome HELLP. (Rojas, Gerardo, Cantillo, \& Cantillo, 2015)

Al comparar ambos estudios se determinó definitivamente que la enfermedad crónica más presentadas en las mujeres embarazadas es la Insuficiencia renal Crónica, las alteraciones renales agudas se presenta comúnmente en las mujeres embarazadas el cual pueden manifestarse a un largo plazo de los tres trimestres del embarazo, causando principalmente hemorragias.

También en el presente estudio se determinó el número de embarazos de las mujeres con enfermedades crónicas, donde la gran mayoría fue su tercer embarazo abarcando un porcentaje del 34,04\%, el cual el año de padecimiento de las pacientes con la enfermedades crónicas correspondían de 0 a 3 años con un el 63,83\%.

Según en el estudio de Iván García Alonso realizado en el año 2016 con el tema "Riesgos del embarazo en la edad avanzada" fue de tipo retrospectivo y analítico de casos y controles sobre los riesgos del embarazo en las gestantes de 35 y más años, donde el número de embarazos en el que se encontraban las pacientes fueron su 2do parto abarcando un 61,90\%. (García Alonso \& Alemán Mederos, 2016)

En relación al presente estudio se pudo observar una gran diferencia ya que en el estudio de Iván García más de la mitad de las mujeres se encontraban en su segundo embarazo mientras que en el presente estudio las mujeres estaban en su tercer embarazo, el embarazo en la edad avanzada tiene mayores riesgos, el cual tiende a desarrollar enfermedades crónicas como las que se encontraron en el grupo estudio del presente trabajo investigativo.

En este estudio se determinó que las complicaciones médicas frecuentes en las mujeres embarazadas fue el síndrome HELLP con un 38,3\%, seguida de la preeclampsia severa con un $31,9 \%$.

Según Maricela Carolina Bajaña Quinto, realizó un estudio en el 2018 con el tema “Asociación de la edad materna con las complicaciones gestacionales", un estudio observacional, analítico y transversal, el cual se llevó a cabo en el departamento de obstetricia del Hospital Vicente Corral Moscoso, de la ciudad de Cuenca de Ecuador, la población fue de 729 pacientes con la ayuda de la revisión de historia clínica, el cual la complicación médica de las embarazadas con mayor frecuencia fue la preeclampsia con un 36,4\%, la hemorragia al postparto el 25,6\%, aborto 17,3\%, hipertensión arterial 7,2\% entre otras. (Bajana Quinto, y otros, 2018)

Al analizar el estudio de Bajaña Quinto se constató que las complicaciones dadas en relación al presente trabajo investigativo fue diferentes, ya que en dicho estudio reflejo que fue la preeclampsia y la hemorragia al postparto las que más presentaron, mientras que en el presente estudio fue el síndrome HELLP, sin embargo la preeclampsia ocupa un segundo lugar, el cual es de suma importancia recalcar que la preeclampsia es una enfermedad frecuente y potencialmente grave que aumenta el riesgo de morbilidad de la madre.

La consecución de lo antes dicho se basa en los siguientes pilares: diagnóstico oportuno de la 
EC, evaluación preconcepcional de la enferma, estratificación del riesgo materno-fetal, optimización hemodinámica, administración de medicamentos seguros a las menores dosis posibles, consejería reproductiva y establecimiento de pronóstico de la madre a largo plazo. (Ovalle, 2014)

El manejo multidisciplinario de las pacientes con alto riesgo obstétrico ofrece la posibilidad de mitigar las complicaciones derivadas de la misma; por lo que se recomienda transferirlas de forma temprana a centros especializados con atención a este tipo de pacientes (Ferrer \& Garzón, 2016).

Por otro lado, se identificó si las mujeres embarazadas han utilizado anticonceptivos el cual el $29,79 \%$ de las pacientes no han utilizado, sin embargos el 21,26\% ha empleado la inyección como método anticonceptivo alternativo, seguido del 14,89\% por uso de pastillas.

Según el estudio de Electra A. González realizado en el año 2017 con el tema "Determinantes en la elección de anticonceptivos en adolescentes consultantes en un centro de atención de salud sexual y reproductiva", fue de tipo transversal analítico. Como herramienta de recolección de daos utilizó un cuestionario anónimo y auto-administrado y fue aplicado a 116 mujeres que consultaron durante un año a un centro de salud sexual y reproductiva, el cual una de las preguntas de suma importancia fue el método anticonceptivo más utilizados en las pacientes, donde reflejó que fue el método inyectable con un $68,1 \%$, la píldora un $37,1 \%$, preservativos masculino un 19\%, implante subcutáneo un 6\%, DIU un 4,3\%, parche un 0,9\% y Anillo vaginal un $0,9 \%$. Dando a conocer que dicho estudio el método inyectable fue el más frecuente. (González, Breme, González, Molina, \& Leal, 2017)

Entonces se dio a conocer que en el estudio de González al igual que el presente estudio el método inyectable fue el más usado seguido de la píldora.

\section{Conclusión}

- Se identificó que uno de los métodos anticonceptivos más utilizados como prevención en las complicaciones por enfermedades crónicas asociadas al embarazo en las pacientes atendidas en el hospital Teodoro Maldonado Carbo fue el método inyectable seguido de las pastillas anticonceptivas, Sin embargo, un gran porcentaje no utilizaron estos métodos generando a largo plazo complicaciones y riegos durante el parto, convirtiéndose en sí ya un problema de salud pública.

- Las características sociodemográficas de las mujeres embarazadas con enfermedades crónicas que acudieron al Hospital Teodoro Maldonado Carbo la gran mayoría abarcaba un rango de edad de 35 a 44 años, con un nivel de instrucción de secundaría, de grupo étnico mestiza y su lugar de residencia en la zona urbana de la ciudad de Guayaquil, dentro de esta población estudiada, se reconoce que el nivel de conocimiento sobre los métodos anticonceptivos fue regular, lo cual influye de manera importante ante las complicaciones por enfermedades crónicas.

- Se determinó que las enfermedades crónicas asociadas al embarazo de las pacientes más frecuentes fue la insuficiencia renal crónica en hemodiálisis y la Hipertensión arterial Crónica presentando a la vez complicaciones médicas como el síndrome de HELLP y preeclampsia severa, los cuales se convierte un gran problema de salud pública ya que genera gastos familiares y del hospital, las enfermedades crónicas llevan mucho tiempo de tratamiento, algunos casos no tienen cura, generando discapacidades residuales y que afectan severamente a la calidad de vida de la paciente. 
- Se verificó mediante la historia clínica si el médico ha recomendado a la tratante utilizar métodos anticonceptivos, la gran mayoría se observó que no lo hacía, ya que se sabe que los métodos anticonceptivos reduce la posibilidad de tener un embarazo en mujeres con enfermedades crónicas.

\section{Referencias Bibliográficas}

Adarve Hidalgo, E., Falguera, G., \& Seguranyes, G. (2016). Adherencia y cumplimiento del método anticonceptivo hormonal oral. Matronas Prof, 17(1), 28-34. Obtenido de www.federacion-matronas.org/wp-content/uploads/2018/01/revbibliografiaadherencia-y-cumplimiento.pdf

Bajana Quinto, M. C., Carrasco Guevara, A. J., Zapata Toapanta, A. E., Ramírez Santana, K. E., Cuadro Terán, R. E., Ramírez Santana, M. E., \& Cordones Sevillano, M. A. (2018). Asociación de la edad materna con las complicaciones gestacionales. Revista Latinoamericana de Hipertensión, 13(2), 52-57. Obtenido de http://www.revhipertension.com/rlh_2_2018/8_asociacion_de_la_edad_materna.pdf

Braganza Guananga, I. (2016). Diseño de una estrategia de intervención educativa sobre métodos anticonceptivos en mujeres en edad fértil. San Francisco La Providencia, San José de Tanquis. Columbe, enero a junio 2016. Chimborazo: Bachelor's thesis, Escuela Superior Politécnica de Chimborazo. Obtenido de http://dspace.espoch.edu.ec/bitstream/123456789/5940/1/10T00151.pdf

Calixto Morales, D., Ospina Díaz, J., \& Manrique Abril, F. (2015). Retiro temprano del implante subdérmico con etonogestrel en usuarias de un programa de anticoncepción de Tunja - Boyacá, Colombia. Universidad y Salud, 17(2), 224-232. Obtenido de http://www.scielo.org.co/scielo.php?script=sci_arttext\&pid=S0124-

$71072015000200008 \& \operatorname{lng}=$ en\&tlng=

Escobar González, E. (2016). Diseño de estrategia de intervención educativa sobre anticoncepción hormonal en mujeres en edad fértil no controladas. Chimborazo: Politécnica de Chimborazo. Comunidad Mercedes Cadena. Obtenido de http://dspace.espoch.edu.ec/handle/123456789/5950

Fawed Reyes, O., Erazo Coello, A., Carrasco Medrano, J. C., Gonzales, D., Mendoza Talavera, A. F., Mejía Rodríguez, M. E., . . . García Fuentes, A. M. (2016). Complicaciones Obstétricas en Adolescentes y Mujeres Adultas con o sin Factores de Riesgo Asociados, Honduras 2016. Archivos de Medicina, 12(4), 1-7. Obtenido de http://www.archivosdemedicina.com/medicina-de-familia/complicaciones-

obsteacutetricas-en-adolescentes-y-mujeres-adultas-con-o-sin-factores-de-riesgoasociados-honduras-2016.pdf

Ferrer, L., \& Garzón, D. (2016). Manejo de paciente obstétrica a término con fibrilación auricular con respuesta ventricular rápida sin inestabilidad hemodinámica. Rev. colomb. anestesiol., $\quad 37(1), \quad 81-83 . \quad$ Obtenido de http://www.scielo.org.co/scielo.php?script=sci_arttext\&pid=S0120$33472009000100011 \& \operatorname{lng}=$ en.

García Alonso, I., \& Alemán Mederos, M. M. (2016). Riesgos del embarazo en la edad avanzada. Revista Cubana de Obstetricia y Ginecología, 39(2), 120-127. Obtenido de http://scielo.sld.cu/pdf/gin/v39n2/gin07213.pdf 
Gómez Sánchez, P., Gaitán Duarte, H., \& Rodríguez Malagón, N. (2017). Adherencia a anticoncepción hormonal combinada (inyectable y oral) en mujeres de 14 a 24 años de edad en 6 ciudades de Colombia. Revista de la Federación Centroamericana de Obstetricia y Ginecología, $\quad 22(4), \quad 77-83 . \quad$ Obtenido de http://www.revcog.org/index.php/revcog/article/viewFile/731/640

González, E. A., Breme, P., González, D. A., Molina, T. G., \& Leal, I. F. (2017). Determinantes en la elección de anticonceptivos en adolescentes consultantes en un centro de atención de salud sexual y reproductiva. Revista chilena de obstetricia y ginecología, 82(6), 692-705. Obtenido de https://scielo.conicyt.cl/pdf/rchog/v82n6/0048-766X-rchog-82-06-0692.pdf

Huerta, M., Sáinz Gómez, J., \& Antonio Solís, J. (2014). Perfil socioeconómico y demográfico de las usuarias del Programa de Planificación Familiar de la SSA. Salud Pública de México,, 18(2), 321-335. Obtenido de http://saludpublica.mx/index.php/spm/article/view/1308

INEC. (2018). Ecuador en cifras. Quito,Ecuador: Instituto Nacional de Estadisticas y Censos. Obtenido de http://www.ecuadorencifras.gob.ec/en-27-anos-tasa-de-natalidad-disminuyoen-134-nacidos-

Mera Zambrano, A., Roca Castro, Y., \& Medranda Obregón, E. (2016). Salud sexual y reproductiva en las mujeres de los cantones Montecristi, Jaramijó y Manta (Ecuador). Margen, 79(2).

Ministerio de Salud Pública. (2017). Plan Nacional de Salud Sexual y Salud Reproductiva 2017-2021. Quito: República del Ecuador. Obtenido de http://repositorio.utn.edu.ec/bitstream/123456789/2010/2/guia\%20535.pdf

OMS. (2016). Plan operacional para llevar adelante la Estrategia Mundial para la Salud de la Mujer, el Niño y el Adolescente. Ginebra: Organzación Mundial de la Salud.

Ovalle, A. K. (2014). Factores asociados con el parto prematuro entre 22 y 34 semanas en un hospital público de Santiago. Revista Médica de Chile., 140(1), 19-29. Obtenido de https://doi.org/10.4067/S0034-98872012000100003

Pozuelos Mendoza, J. (2018). Anticonceptivos hormonales y endometrio. Revista de la Federación Centroamericana de Obstetricia y Ginecología, 2(14), 19-23. Obtenido de www.revistamedica.org/index.php/revcog/article/download/525/434

Rojas, Gerardo, W., Cantillo, J. d., \& Cantillo, M. J. (2015). Enfermedad renal crónica y embarazo. Acta Médica Colombiana, 40(4), 331-337. Obtenido de scielo.org.co/pdf/amc/v40n4/v40n4a11.pdf 


\section{PARA CITAR EL ARTÍCULO INDEXADO.}

Sánchez Coronel, N. C., Suarez Lima, G. J. S. L., Holguín Salazar, N. F., \& Zuñiga Fariño, L. J. (2020). Anticoncepción como prevención de complicaciones por enfermedades crónicas $\begin{array}{llll}\text { asociadas al embarazo. } & \text { ConcienciaDigital, } & \text { 3(2.2), }\end{array}$ https://doi.org/10.33262/concienciadigital.v3i2.2.1243

\section{Ciencia \\ LDigital}

El artículo que se publica es de exclusiva responsabilidad de los autores y no necesariamente reflejan el pensamiento de la Revista Conciencia Digital.

El artículo queda en propiedad de la revista y, por tanto, su publicación parcial y/o total en otro medio tiene que ser autorizado por el director de la Revista Conciencia Digital.
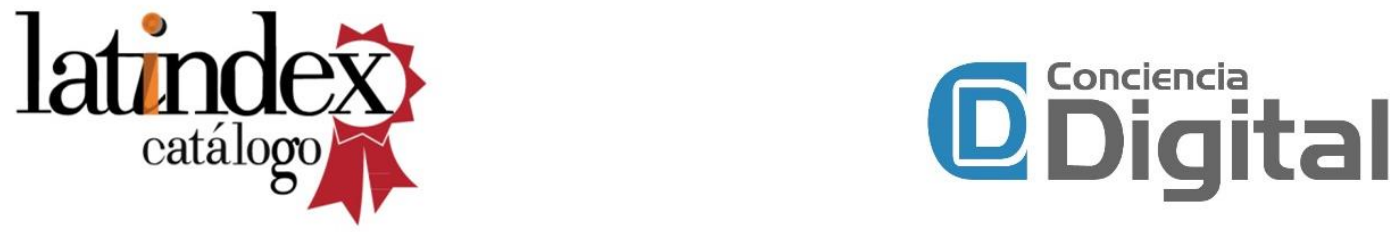